\section{CPS-274 REAL WORLD EFFECTIVENESS OF PALBOCICLIB AND RIBOCICLIB IN WOMEN WITH METASTATIC BREAST CANCER}

${ }^{1} \mathrm{M}$ García-Trevijano Cabetas*, ${ }^{1} \mathrm{P}$ Lucena, ${ }^{1} \mathrm{I}$ Jiménez-Nácher, ${ }^{1} \mathrm{E}$ Villamañán, ${ }^{1} \mathrm{C}$ Sobrino ${ }^{1} \mathrm{C}$ Bilbao, ${ }^{2} \mathrm{P}$ Zamora, ${ }^{1} \mathrm{~A}$ Herrero. ${ }^{1}$ La Paz University Hospital, Hospital Pharmacy Department, Madrid, Spain; ${ }^{2}$ La Paz University Hospital, Oncology Department, Madrid, Spain

\subsection{6/ejhpharm-2021-eahpconf.106}

Background and importance Palbociclib and ribociclib are novel oral agents in hormone receptor positive $(\mathrm{HR}+)$ and human epidermal growth factor 2 negative (HER2-) metastatic breast cancer (MBC). As these drugs have recently been released, it is necessary to provide insight into their real world use.

Aim and objectives The aim of this study was to provide data on effectiveness in patients treated with palbociclib and ribociclib in clinical practice.

Material and methods This observational retrospective study was performed in a tertiary hospital and included $\mathrm{HR}+/$ HER2- MBC patients who initiated treatment with palbociclib or ribociclib (March 2018 to March 2019). Patients were followed-up until Marchc2020. Patient demographics, clinical characteristics and treatment patterns were obtained from electronic medical records and the pharmacy database, Farmatools. The primary effectiveness variable was progression free survival (PFS). Overall survival (OS), and survival probabilities at 12 and 18 months were also estimated. OS was estimated with the Kaplan-Meier test and PFS with a competitive risk study, using the software R (V.2013).

Results 61 patients were enrolled: 33 treated with palbociclib and 28 with ribociclib. Median follow-up was 12.2 and 15.2 months in the palbociclib and ribociclib groups, respectively. Median age was 59 years (40-86). Eastern Cooperative Oncology Group Performance Status (ECOG PS) was 0-1 in most patients. Palbociclib was mainly used as secondline $(n=27$; $81.8 \%)$ and ribociclib as firstline $(n=19 ; 67.9 \%)$ treatment. Treatment was discontinued in 24 patients $(72.7 \%)$ receiving palbociclib and in 12 patients (42.9\%) receiving ribociclib. Disease progression was the most common reason for discontinuation: 21 patients $(63.6 \%)$ receiving palbociclib and 9 patients (32.1\%) receiving ribociclib. In the palbociclib group, median PFS was 12.7 months (95\% CI 7.5 to not estimable) and the 12 month and 18 month PFS rates were $51.5 \%$ (95\% CI 34 to 69 ) and $37.7 \%$ (95\% CI 20.1 to 55.4 ), respectively. Median duration of PFS was not reached in the ribociclib group; the 12 month and 18 month PFS rates were $78.6 \%$ (95\% CI 63 to 94.1) and 68.9\% (95\% CI 49.9 to 88 ), respectively. OS outcomes were not mature at data cut off, with eight deaths in patients receiving palbociclib and four in those receiving ribociclib. The 12 month and 18 month OS rates were $87.7 \%$ (SE 6.8\%) and 61.6\% (SE $12.2 \%$ ) in the Palbociclib group and $95.8 \%$ (SE 4.1\%) and $87.1 \%$ (SE 7\%) in the ribociclib group.

Conclusion and relevance Our findings in the real world setting confirmed the clinical benefit for women with $\mathrm{HR}+$ / HER2 - MBC. Palbociclib and ribociclib outcomes were comparable with those reached in the phase III trials, PALOMA-3 and MONALEESA-2, due to the profile of the patients treated with both drugs. As palbociclib and ribociclib were used in different settings, outcomes cannot be compared.
Conflict of interest No conflict of interest

\section{CPS-275 EFFICACY AND SAFETY OF TRIFLURIDINE/TIPIRACIL IN PATIENTS WITH METASTATIC COLORECTAL CANCER: REAL WORLD DATA}

I Patier*, FF Fernando, MG Maria, DF Raul, MG Teresa. Hospital Universitario De Getafe, Pharmacy, Getafe, Spain

\subsection{6/ejhpharm-2021-eahpconf.107}

Background and importance Trifluridine/tipiracil (TAS-102) is a drug with a doubtful clinical benefit according to the ESMOMCBS scale (values=1-2) in the treatment of patients with metastatic colorectal cancer (mCRC) who have previously been treated with, or are not considered candidates for, available therapies, including fluoropyrimidine, oxaliplatin and irinotecan based chemotherapies, anti-VEGF agents and antiEGFR agents.

Aim and objectives The objective of this study was to evaluate the effectiveness and safety of TAS-102 in mCRC in real world use.

Material and methods An observational, retrospective, descriptive study was conducted in all patients with a diagnosis of mCRC who received treatment with TAS-102 from January 2017 to April 2019. Demographic (sex and age) and clinical variables (RAS gene mutation, primary tumour location, duration of treatment, progression free survival (PFS) and adverse events) were analysed. Progression was analysed according to the response evaluation criteria in solid tumours (RECIST V.1.1).

Results 32 patients with mCRC were included. Mean age was 69.4 years (IQR 61.7-75.4). RAS wild-type was detected in $53.1 \%$ of patients. The primary location was the colon in $71.9 \%$ (43.5\% unspecified, 30.5\% left, $26 \%$ right), the rectum in $25 \%$ and both in $3.1 \% .3 .1 \%$ of patients received two previous lines of treatment, $68.8 \%$ three and $28.1 \%$ four (schemes with capecitabine \pm oxaliplatin, FOLFOX, FOLFIRI and regorafenib). The addition of anti-VEGFR/EGFR therapy was not considered a differentiated scheme.

All patients progressed to fluoropyrimidines, $96.9 \%$ progressed to irinotecan and $53.1 \%$ to cetuximab. $100 \%$ of patients received biological therapy. Bevacizumab was administered to $90.6 \%$ of patients, aflibercept to $59.4 \%$ and cetuximab/panitumumab to $53.1 \%$. Mean duration of treatment (months) was 2.3 (IQR1.6-3.3). At the time of data collection, $81.2 \%$ had progressed, $12.5 \%$ stopped because of toxicity and $6.2 \%$ remained on the regimens. Median PFS was $2.3(95 \%$ CI 1.5 to 3.0$)$ months. Any grade adverse events occurred in $90.6 \%$ of patients. These were asthenia (65.6\%), pain (56.2\%), neutropenia (40.6\%; differentiated as $38.5 \%$ grade 3 , $46.1 \%$ grade $2,15.4 \%$ grade 1 ), diarrhoea (34.4\%), nausea $(31.3 \%)$, anorexia (18.7\%), liver toxicity $(18.7 \%)$, infections (15.6\%) and haemorrhages (9.4\%).

Conclusion and relevance In our study, patients treated with trifluridine/tipiracil presented toxicity and a PFS similar to that observed in the pivotal clinical trial (2.0 (1.9 to 2.1)). TAS-102 is a low clinical benefit option in mCRC.

\section{REFERENCES AND/OR ACKNOWLEDGEMENTS}

Conflict of interest No conflict of interest 